\title{
On Absolute Matrix Summability Factors of Infinite Series and Fourier Series
}

\section{Şebnem YILDIZ ${ }^{1}$}

${ }^{I}$ Department of Mathematics, Ahi Evran University, Kırşehir, Turkey.

\begin{tabular}{l} 
Article Info \\
\hline Received: 08/10/2016 \\
Accepted: 10/11/2016 \\
Keywords \\
\hline Absolute matrix \\
summability \\
Fourier series \\
Infinite series \\
Hölder inequality \\
Minkowski inequality
\end{tabular}

\section{INTRODUCTION}

Let $\sum a_{n}$ be a given infinite series with the partial sums $\left(s_{n}\right)$ and $\left(p_{n}\right)$ be a sequence of positive numbers such that

$$
P_{n}=\sum_{v=0}^{n} p_{v} \rightarrow \infty, n \rightarrow \infty,\left(P_{-i}=p_{-i}=0, i \geq 1\right)
$$

The sequence -to-sequence transformation

$$
T_{n}=\frac{1}{P_{n}} \sum_{v=0}^{n} p_{v} s_{v}
$$

defines the sequence $\left(T_{n}\right)$ of the Riesz mean or simply the $\left(\bar{N}, p_{n}\right)$ mean of the sequence $\left(s_{n}\right)$ generated by the sequence of coefficients $\left(p_{n}\right)$ (see [7]). The series $\sum a_{n}$ is said to be summable $\left|\bar{N}, p_{n}\right|_{k}, k \geq 1$, if (see [1])

$$
\sum_{n=1}^{\infty}\left(\frac{P_{n}}{p_{n}}\right)^{k-1}\left|T_{n}-T_{n-1}\right|^{k}<\infty .
$$

In the special case when $p_{n}=1$ for all values of $\mathrm{n}($ resp. $k=1),\left|\bar{N}, p_{n}\right|_{k}$ summability is the same as $|C, 1|_{k}$ (resp.| $\left.\bar{N}, p_{n} \mid\right)$ summability. 
Let $f$ be a periodic function with period $2 \pi$ and Lebesgue integrable over $(-\pi, \pi)$. Without any loss of generality we may assume that the constant term in the Fourier series of $f(t)$ is zero, so that

$$
\int_{-\pi}^{\pi} f(t) d t=0
$$

and

$$
f(t) \sim \sum_{n=1}^{\infty}\left(a_{n} \cos n t+b_{n} \sin n t\right)=\sum_{n=1}^{\infty} C_{n}(t)
$$

We write

$$
\phi(t)=\frac{1}{2}\{f(x+t)+f(x-t)\}, \quad \phi_{1}(t)=\frac{1}{t} \int_{0}^{t} \phi(u) d u .
$$

Given a normal matrix $A=\left(a_{n v}\right)$, we associate two lower semimatrices $\bar{A}=\left(\bar{a}_{n v}\right)$ and $\hat{A}=\left(\hat{a}_{n v}\right)$ as follows:

$$
\bar{a}_{n v}=\sum_{i=v}^{n} a_{n i}, \quad n, v=0,1, \ldots \quad \bar{\Delta} a_{n v}=a_{n v}-a_{n-1, v}, \quad a_{-1,0}=0
$$

and

$$
\hat{a}_{00}=\bar{a}_{00}=a_{00}, \quad \hat{a}_{n v}=\bar{\Delta} \bar{a}_{n v}, \quad n=1,2, \ldots
$$

It may be noted that $\bar{A}$ and $\hat{A}$ are the well-known matrices of series-to-sequence and series-to-series transformations, respectively. Then, we have

$$
\begin{gathered}
A_{n}(s)=\sum_{v=0}^{n} a_{n v} s_{v}=\sum_{v=0}^{n} \bar{a}_{n v} a_{v} \\
\bar{\Delta} A_{n}(s)=\sum_{v=0}^{n} \hat{a}_{n v} a_{v} .
\end{gathered}
$$

Let $\left(\theta_{n}\right)$ be any sequence of positive real numbers. The series $\sum a_{n}$ is said to be summable $\left|A, \theta_{n}\right|_{k}$, $k \geq 1$, if (see [10])

$$
\sum_{n=1}^{\infty} \theta_{n}^{k-1}\left|\bar{\Delta} A_{n}(s)\right|^{k}<\infty
$$

where

$$
\bar{\Delta} A_{n}(s)=A_{n}(s)-A_{n-1}(s) .
$$


Remark. If we take $\theta_{n}=\frac{P_{n}}{p_{n}}$, then $\left|A, \theta_{n}\right|_{k}$ summability reduces to $\left|A, p_{n}\right|_{k}$ summability (see [11]). Also, if we take $\theta_{n}=\frac{P_{n}}{p_{n}}$ and $a_{n v}=\frac{p_{v}}{P_{n}}$, then we get $\left|\bar{N}, p_{n}\right|_{k}$ summability. Furthermore, if we take $\theta_{n}=n, a_{n v}=\frac{p_{v}}{P_{n}}$ and $p_{n}=1$ for all values of $\mathrm{n}$, then $\left|A, \theta_{n}\right|_{k}$ reduces to $|C, 1|_{k}$ summability (see [6]). Finally, if we take $\theta_{n}=n$ and $a_{n v}=\frac{p_{v}}{P_{n}}$, then we get $\left|R, p_{n}\right|_{k}$ summability (see [3]).

\section{THE KNOWN RESULTS}

The following theorems are known dealing with Fourier series (see [2]).

Theorem 2.1. Let $\left(p_{n}\right)$ be a sequence of positive numbers such that

$$
\begin{gathered}
P_{n}=O\left(n p_{n}\right) \text { as } n \rightarrow \infty, \\
P_{n} \Delta p_{n}=O\left(p_{n} p_{n+1}\right) .
\end{gathered}
$$

If $\phi_{1}(t)$ is of bounded variation in $(0, \pi)$ and $\left(\lambda_{n}\right)$ is a sequence such that

$$
\sum_{n=1}^{\infty} \frac{1}{n}\left|\lambda_{n}\right|^{k}<\infty
$$

and

$$
\sum_{n=1}^{\infty}\left|\Delta \lambda_{n}\right|<\infty
$$

then the series $\sum C_{n}(t) \frac{\lambda_{n} P_{n}}{n p_{n}}$ is summable $\left|\bar{N}, p_{n}\right|_{k}, k \geq 1$.

Theorem 2.2. If the sequences $\left(p_{n}\right)$ and $\left(\lambda_{n}\right)$ satisfy the conditions (12)-(15) of Theorem 2.1 and

$$
B_{n} \equiv \sum_{v=1}^{n} v a_{v}=O(n), \quad n \rightarrow \infty
$$

then the series $\sum a_{n} \frac{\lambda_{n} P_{n}}{n p_{n}}$ is summable $\left|\bar{N}, p_{n}\right|_{k}, k \geq 1$

\section{THE MAIN RESULTS}

Many studies have been done for Riesz summability and matrix generalization of infinite series and Fourier series (see [4], [5], [9], [12]). The aim of this paper is to generalize Theorem 2.1 and Theorem 2.2 under suitable and different conditions using general summability factors for $\left|A, \theta_{n}\right|_{k}$ summability methods.

Now, we shall prove the following theorems. 
Theorem 3.1. Let $A=\left(a_{n v}\right)$ be a positive normal matrix such that

$$
\begin{gathered}
\bar{a}_{n 0}=1, \quad n=0,1, \ldots, \\
a_{n-1, v} \geq a_{n v}, \text { for } n \geq v+1, \\
a_{n n}=O\left(\frac{p_{n}}{P_{n}}\right), \\
\hat{a}_{n, v+1}=O\left(v\left|\bar{\Delta} a_{n v}\right|\right) .
\end{gathered}
$$

Let $\phi_{1}(t)$ be of bounded variation in $(0, \pi)$ and $\left(\theta_{n} a_{n n}\right)$ be a non-increasing sequence. If the conditions (12), (13), (15) of Theorem 2.1 are satisfied and $\left(\theta_{n}\right)$ is any sequence of positive constants such that

$$
\sum_{n=1}^{\infty} \theta_{n}{ }^{k-1} \frac{\left|\lambda_{n}\right|^{k}}{n^{k}}<\infty,
$$

then the series $\sum C_{n}(t) \frac{\lambda_{n} P_{n}}{n p_{n}}$ is summable $\left|A, \theta_{n}\right|_{k}, k \geq 1$.

Theorem 3.2. If the conditions (12), (13) and (15-21) are satisfied and $\left(\theta_{n} a_{n n}\right)$ is a non increasing sequence, then the series $\sum a_{n} \frac{\lambda_{n} P_{n}}{n p_{n}}$ is summable $\left|A, \theta_{n}\right|_{k}, k \geq 1$.

Remark. It should be noted that in the above theorems, if we take $\theta_{n}=\frac{P_{n}}{p_{n}}$ and $a_{n v}=\frac{p_{v}}{P_{n}}$, then we get Theorem 2.1 and Theorem 2.2. In this case, condition (21) reduces to condition (14).

We need the following lemmas for the proof of our theorems.

Lemma 3.3 [8] If $\phi_{1}(t)$ is of bounded variation in $(0, \pi)$, then

$$
\sum_{n=1}^{\infty} v C_{v}(x)=O(n) \quad \text { as } n \rightarrow \infty
$$

Lemma 3.4 [2] If the sequence $\left(p_{n}\right)$ such that conditions (12) and (13) of Theorem 2.1 are satisfied, then

$$
\Delta\left\{\frac{P_{n}}{p_{n} n^{2}}\right\}=O\left(\frac{1}{n^{2}}\right) .
$$

\section{PROOF OF THEOREM 3.2.}

Let $\left(I_{n}\right)$ denotes the A-transform of the series $\sum a_{n} P_{n} \lambda_{n}\left(n p_{n}\right)^{-1}$. Then, by (9) and (10), we have 


$$
\bar{\Delta}_{n}=\sum_{v=1}^{n} \hat{a}_{n v} a_{v} P_{v} \lambda_{v}\left(v p_{v}\right)^{-1} .
$$

Applying Abel's transformation to this sum, we get that

$$
\begin{aligned}
& \bar{\Delta} I_{n}=\sum_{v=1}^{n} \hat{a}_{n v} a_{v} P_{v} \lambda_{v}\left(v p_{v}\right)^{-1} \\
& =\sum_{v=1}^{n-1} \Delta\left(\frac{\hat{a}_{n v} P_{v} \lambda_{v}}{v^{2} p_{v}}\right) \sum_{r=1}^{v} r \cdot a_{r}+\frac{\hat{a}_{n n} P_{n} \lambda_{n}}{n^{2} p_{n}} \sum_{r=1}^{n} r \cdot a_{r} \\
& =\left\{\sum_{v=1}^{n-1} \frac{\bar{\Delta} a_{n v} P_{v} \lambda_{v}}{v^{2} p_{v}}+\sum_{v=1}^{n-1} \frac{\hat{a}_{n, v+1} P_{v}}{v^{2} p_{v}} \Delta \lambda_{v}+\sum_{v=1}^{n-1} \hat{a}_{n, v+1} \lambda_{v+1} \Delta\left(\frac{P_{v}}{v^{2} p_{v}}\right)\right\} \sum_{r=1}^{v} r \cdot a_{r}+\frac{a_{n n} \lambda_{n} P_{n}}{n^{2} p_{n}} \sum_{r=1}^{n} r \cdot a_{r} \\
& =\frac{a_{n n} \lambda_{n} P_{n}}{n^{2} p_{n}} B_{n}+\sum_{v=1}^{n-1} \frac{\bar{\Delta} a_{n v} P_{v} \lambda_{v}}{v^{2} p_{v}} B_{v}+\sum_{v=1}^{n-1} \hat{a}_{n, v+1} \lambda_{v+1} \Delta\left(\frac{P_{v}}{v^{2} p_{v}}\right) B_{v}+\sum_{v=1}^{n-1} \frac{\hat{a}_{n, v+1} P_{v}}{v^{2} p_{v}} \Delta \lambda_{v} B_{v} \\
& =I_{n, 1}+I_{n, 2}+I_{n, 3}+I_{n, 4} .
\end{aligned}
$$

To complete the proof of Theorem 3.2, by Minkowski inequality, it is sufficient to show that

$$
\sum_{n=1}^{\infty} \theta_{n}{ }^{k-1}\left|I_{n, r}\right|^{k}<\infty, \text { for } r=1,2,3,4
$$

Firstly, we have that

$$
\begin{aligned}
& \sum_{n=1}^{m} \theta_{n}{ }^{k-1}\left|I_{n, 1}\right|^{k}=O(1) \sum_{n=1}^{m} \theta_{n}{ }^{k-1}\left|\frac{a_{n n} \lambda_{n} P_{n}}{n^{2} p_{n}} B_{n}\right|^{k}=O(1) \sum_{n=1}^{m} \theta_{n}{ }^{k-1}\left|\lambda_{n}\right|^{k}\left|B_{n}\right|^{k} \frac{1}{n^{2 k}} \\
& =O(1) \sum_{n=1}^{m} \theta_{n}{ }^{k-1} \frac{\left|\lambda_{n}\right|^{k}}{n^{k}}=O \text { (1) as } m \rightarrow \infty
\end{aligned}
$$

by virtue of the hypotheses of Theorem 3.2. Now, applying Hölder's inequality, we have that

$$
\begin{aligned}
& \sum_{n=2}^{m+1} \theta_{n}{ }^{k-1}\left|I_{n, 2}\right|^{k}=\sum_{n=2}^{m+1} \theta_{n}{ }^{k-1}\left|\sum_{v=1}^{n-1} \bar{\Delta} a_{n v} \frac{P_{v} \lambda_{v}}{v^{2} p_{v}} B_{v}\right|^{k} \\
& =O(1) \sum_{n=2}^{m+1} \theta_{n}{ }^{k-1}\left\{\sum_{v=1}^{n-1} \bar{\Delta} a_{n v}\left(\frac{P_{v}}{v^{2} p_{v}}\right)^{k}\left|\lambda_{v}\right|^{k}\left|B_{v}\right|^{k}\right\} \times\left\{\sum_{v=1}^{n-1}\left|\bar{\Delta} a_{n v}\right|\right\}^{k-1} \\
& =O(1) \sum_{n=2}^{m+1} \theta_{n}{ }^{k-1} a_{n n}{ }^{k-1} \sum_{v=1}^{n-1}\left|\bar{\Delta} a_{n v}\right|\left(\frac{P_{v}}{v^{2} p_{v}}\right)^{k}\left|\lambda_{v}\right|^{k}\left|B_{v}\right|^{k}
\end{aligned}
$$




$$
\begin{aligned}
& =O(1) \sum_{v=1}^{m}\left|\lambda_{v}\right|^{k}\left|B_{v}\right|^{k}\left(\frac{P_{v}}{v^{2} p_{v}}\right)^{k} \sum_{n=v+1}^{m+1}\left(\theta_{n} a_{n n}\right)^{k-1}\left|\bar{\Delta} a_{n v}\right| \\
& =O(1) \sum_{v=1}^{m}\left(\theta_{v} a_{v v}\right)^{k-1}\left|\lambda_{v}\right|^{k}\left|B_{v}\right|^{k}\left(\frac{P_{v}}{v^{2} p_{v}}\right)^{k} \sum_{n=v+1}^{m+1}\left|\bar{\Delta} a_{n v}\right| \\
& =O(1) \sum_{v=1}^{m}\left(\theta_{v} a_{v v}\right)^{k-1} \frac{\left|\lambda_{v}\right|^{k}}{v^{k}}\left(\frac{P_{v}}{p_{v}}\right)^{k-1}=O(1) \sum_{v=1}^{m} \theta_{v}^{k-1} \frac{\left|\lambda_{v}\right|^{k}}{v^{k}}=O(1) \text { as } m \rightarrow \infty,
\end{aligned}
$$

by virtue of the hypotheses of Theorem 3.2. On the other hand, since $\Delta\left\{\frac{P_{v}}{v^{2} p_{v}}\right\}=O\left(\frac{1}{v^{2}}\right)$ by Lemma 3.4, we obtain

$$
\begin{aligned}
& \sum_{n=2}^{m+1} \theta_{n}{ }^{k-1}\left|I_{n, 3}\right|^{k}=\sum_{n=2}^{m+1} \theta_{n}{ }^{k-1}\left|\sum_{v=1}^{n-1}\right| \hat{a}_{n, v+1} \| \lambda_{v+1}\left|\Delta\left(\frac{P_{v}}{v^{2} p_{v}}\right) B_{v}\right|^{k} \\
& =O(1) \sum_{n=2}^{m+1} \theta_{n}{ }^{k-1}\left\{\sum_{v=1}^{n-1}\left|\hat{a}_{n, v+1}\right|\left|\lambda_{v+1}\right|^{k} \frac{1}{v}\right\} \times\left\{\sum_{v=1}^{n-1} \frac{\left|\hat{a}_{n, v+1}\right|}{v}\right\}^{k-1} \\
& =O(1) \sum_{n=2}^{m+1} \theta_{n}{ }^{k-1}\left\{\sum_{v=1}^{n-1}\left|\hat{a}_{n, v+1}\right|\left|\lambda_{v+1}\right|^{k} \frac{1}{v}\right\} \times\left\{\sum_{v=1}^{n-1}\left|\bar{\Delta} a_{n v}\right|\right\}^{k-1} \\
& =O(1) \sum_{v=1}^{m}\left|\lambda_{v+1}\right|^{k} \frac{1}{v_{n=v+1}} \sum_{n}^{m+1}\left(\theta_{n} a_{n n}\right)^{k-1}\left|\hat{a}_{n, v+1}\right|=O(1) \sum_{v=1}^{m}\left(\theta_{v} a_{v v}\right)^{k-1}\left|\lambda_{v+1}\right|^{k} \frac{1}{v+1}\left(1+\frac{1}{v}\right) \\
& =O(1)\left(\theta_{1} a_{11}\right)^{k-1} \sum_{v=1}^{m}\left|\lambda_{v+1}\right|^{k} \frac{1}{v}=O(1) \text { as } m \rightarrow \infty,
\end{aligned}
$$

by virtue of the hypotheses of Theorem 3.2 and Lemma 3.3. Finally, we get

$$
\begin{aligned}
& \sum_{n=2}^{m+1} \theta_{n}{ }^{k-1}\left|I_{n, 4}\right|^{k}=\sum_{n=2}^{m+1} \theta_{n}{ }^{k-1}\left|\sum_{v=1}^{n-1} \frac{\hat{a}_{n, v+1} P_{v}}{v^{2} p_{v}} \Delta \lambda_{v} B_{v}\right|^{k} \\
& =O(1) \sum_{n=2}^{m+1} \theta_{n}{ }^{k-1}\left|\sum_{v=1}^{n-1} \frac{\hat{a}_{n, v+1}}{v} \Delta \lambda_{v} B_{v}\right|^{k} \\
& =O(1) \sum_{n=2}^{m+1} \theta_{n}{ }^{k-1}\left\{\sum_{v=1}^{n-1}\left|\hat{a}_{n, v+1}\right|\left|\Delta \lambda_{v}\right| \frac{\left|B_{v}\right|^{k}}{v^{k}}\right\} \times\left\{\sum_{v=1}^{n-1}\left|\hat{a}_{n, v+1}\right|\left|\Delta \lambda_{v}\right|\right\}^{k-1} \\
& =O(1) \sum_{n=2}^{m+1} \theta_{n}{ }^{k-1} a_{n n}{ }^{k-1}\left\{\sum_{v=1}^{n-1}\left|\hat{a}_{n, v+1}\right|\left|\Delta \lambda_{v}\right| \frac{\left|B_{v}\right|^{k}}{v^{k}}\right\} \times\left\{\sum_{v=1}^{n-1}\left|\Delta \lambda_{v}\right|\right\}^{k-1}
\end{aligned}
$$




$$
\begin{aligned}
& =O(1) \sum_{v=1}^{m}\left|\Delta \lambda_{v}\right| \frac{\left|B_{v}\right|^{k}}{v^{k}} \sum_{n=v+1}^{m+1}\left(\theta_{n} a_{n n}\right)^{k-1}\left|\hat{a}_{n, v+1}\right| \\
& =O(1) \sum_{v=1}^{m}\left(\theta_{v} a_{v v}\right)^{k-1}\left|\Delta \lambda_{v}\right| \sum_{n=v+1}^{m+1}\left|\hat{a}_{n, v+1}\right| \\
& =O(1)\left(\theta_{1} a_{11}\right)^{k-1} \sum_{v=1}^{m}\left|\Delta \lambda_{v}\right|=O(1) \text { as } m \rightarrow \infty,
\end{aligned}
$$

by virtue of the hypotheses of Theorem 3.2.

This completes the proof of Theorem 3.2.

Proof of Theorem 3.1. Theorem 3.1 is a direct consequence of Theorem 3.2 and Lemma 3.3.

\section{CONCLUSIONS}

If we take $\theta_{n}=\frac{P_{n}}{p_{n}}$ in Theorem 3.1 and Theorem 3.2, then we get two theorems dealing with $\left|A, p_{n}\right|_{k}$ summability (see [13]). Also, if we take $\theta_{n}=\frac{P_{n}}{p_{n}}$ and $a_{n v}=\frac{p_{v}}{P_{n}}$, then we get Theorem 2.1 and Theorem 2.2. Additionally, if we take $\theta_{n}=n$ and $a_{n v}=\frac{p_{v}}{P_{n}}$, then we get a theorem dealing with $\left|R, p_{n}\right|_{k}$ summability. Finally, if we take $\theta_{n}=n, a_{n v}=\frac{p_{v}}{P_{n}}$ and $p_{n}=1$ for all values of $n$, then we get a result for $|C, 1|_{k}$ summability.

\section{CONFLICT OF INTEREST}

No conflict of interest was declared by the authors

\section{REFERENCES}

[1] Bor H., "On two summability methods", Math. Proc. Cambridge Philos Soc., 97, 147149, (1985).

[2] Bor H., "Multipliers for $\left|\bar{N}, p_{n}\right|_{k}$ summability of Fourier series", Bull. Inst. Math. Acad. Sinica, 17, 285-290, (1989).

[3] Bor H., "On the relative strength of two absolute summability methods", Proc. Amer. Math. Soc., 113, 1009-1012, (1991).

[4] Bor H., "Some new results on infinite series and Fourier series", Positivity, 19, 467-473, (2015).

[5] Bor H., "Some new results on absolute Riesz summability of infinite series and Fourier series", Positivity, 20, 599-605, (2016).

[6] Flett T. M., " On an extension of absolute summability and some theorems of Littlewood and Paley", Proc. London Math. Soc., 7, 113-141, (1957). 
[7] Hardy G. H., "Divergent Series", Oxford Univ. Press, Oxford, (1949).

[8] Mishra K. N., "Multipliers for $\left|\bar{N}, p_{n}\right|$ summability of Fourier series", Bull. Inst. Math. Acad. Sinica, 14, 431-438, (1986).

[9] Özarslan H. S. and Yıldız S., "A new study on the absolute summability factors of Fourier series", J. Math. Anal., 7, 31-36, (2016).

[10] Sarıöl M. A., "On the local properties of factored Fourier series", Appl. Math. Comp., 216, 33863390, (2010).

[11] Sulaiman W. T., "Inclusion theorems for absolute matrix summability methods of an infinite series (IV)", Indian J. Pure and Appl. Math., 34 (11), 547-1557, (2003).

[12] Yildız Ş., "A new theorem on local properties of factored Fourier series", Bull Math. Anal. Appl., 8 (2), 1-8, (2016).

[13] Yıldız Ş., " On the absolute matrix summability factors of Fourier series", Math. Notes, (in press). 